\title{
Letter to Editor - Vaccines and Vaccinations
}

\section{DEAR EDITOR}

Given the interest in COVID 19 vaccine; I have attempted to summarize some salient facts about vaccine developments for your Readers.

The year 2020 will be remembered in history for the Covid-19 pandemic. It effected most aspects of our life with far reaching consequences for every section of society. Another aspect of this chaos was the addition of many new words to our vocabulary e.g. social distancing, quarantine, hand hygiene, sanitisers, personal protection equipment, droplet infection, immunity, the latest addition being vaccines and vaccinations!

\section{HISTORY OF VACCINES AND VACCINATIONS}

Many aspects of public health (housing, clean water provision, sanitation, nutrition) have improved significantly over the last couple of centuries. Vaccines too have been refined.

We know; it was the early work of Edward Jenner (1749-1823) which laid the foundation of Immunology. Jenner was a family doctor from Gloucestershire. He made a rational and scientific attempt to control small pox through vaccination. ${ }^{1}$

Louis Pasteur (1822-1895) successfully used the principle of attenuation to develop vaccines against anthrax and rabies. ${ }^{2}$ The same principle was used for vaccines against tuberculosis, measles, mumps and rubella. The problem with the possible mutation of pathogens was overcome by developing vaccines using killed/dead organism; employing either heat or formaldehyde. Vaccines were developed for cholera, pertussis, influenza and tetanus. ${ }^{3}$ Two types of polio vaccines were developed in the mid-20th century. The Jonas Salk's polio vaccine (injectable) was based on formalin-killed poliovirus; while Albert Sabin used an attenuated strain of the pathogen for his oral polio vaccine. ${ }^{4}$

In the late 1940's subunit vaccines were developed, where proteins extracted from pathogens were used to sensitise the vaccinee's immune system against the invading organism. This education and sensitisation of the host's immune system can also be achieved using genetic material (RNA, DNA) extracted from pathogens. ${ }^{5,6}$

\section{TYPES OF VACCINES}

\section{Whole pathogen vaccines}

Using the whole pathogen in the vaccine is a well-known method of vaccination. To overcome the potential side effects of using the pathogen in its natural state, modern vaccines adopt two strategies. The virulent organism used is either in a killed/inactivated state or in an attenuated/ weakened form.

Live/attenuated vaccines require temperaturecontrolled storage; moreover they are not a vaccine of choice in immune compromised individuals. Killed/inactivated vaccines sometimes need the inclusion of immune enhancers/ adjuvants to generate an effective immune response. These are safe for patients with impaired immune response. They usually require booster doses or periodic supplementation for maintenance of the immune response. ${ }^{5}$

\section{Subunit vaccines}

Subunit vaccines consist of components/fragments/ antigens from the pathogen's surface proteins which trigger an immune response. They need inclusion of immune enhancers for generation of a strong and effective host immune response. Genetic coding for the pathogen proteins are passed to the carrier cell which are then able to produce the desired proteins, to be used as an active ingredient of the vaccine itself. ${ }^{7}$ Some bacteria produces toxins; e.g. tetanus is caused by a neurotoxin (tetanospasmin) produced by the bacterium clostridium tetani. The inactivated form of these toxins (toxoids) is used for immunisation; these are known as toxoids vaccines $^{8}$.

Nucleic acid vaccines do not carry any protein antigen to the host body. Using the pathogen's genetic material (either RNA or DNA) they provide the genetic instructions to the relevant cells in the body to produce antigens which in turn trigger an immune response. RNA vaccine uses mRNA (Messenger Ribonucleic Acid). Theoretically these vaccines should produce a strong and effective immune response as the antigens are produced by the host's own cells, but practically human experience with these vaccines is very limited.

Sometimes genetic instructions to the host immune system have been with the help of a harmless virus; these are known as viral vector vaccines. ${ }^{9}$

\section{Coronavirus Vaccines}

The Covid-19 pandemic opened new research platforms in the subject of vaccinology as it was a pandemic. A mammoth effort was launched in various countries to develop an effective vaccine against the coronavirus.

The Pfizer BioNTech vaccine was the first vaccine to be approved for use in adults by WHO (World Health Organisation) and other health regulators. The Pfizer uses synthetic mRNA generated from cDNA (complementary DNA) template copied from viral mRNA. Upon injection into our body, the mRNA directs host cells to make viral proteins (spike proteins occurring on the surface of the virus). These viral proteins are not capable of causing the disease as they are not carried by the whole virus but they do trigger an immune response (antibodies production against the viral proteins and activation T-lymphocytes). The Pfizer BioNTech vaccine must be stored around -70C to maintain its optimal efficacy.

The Oxford University vaccine is a UK vaccine in collaboration with Astra Zeneca. it uses a chimpanzee's adenovirus (a harmless virus causing common cold in chimps). The Oxford vaccine uses this adenovirus as a viral vector. In a similar fashion to the mRNA vaccine, the host raises an immune response to the spike protein producing antibodies and T-lymphocyte activation. The adenovirus has been genetically modified so that it cannot replicate and infect humans. This makes it safe for vulnerable individuals, children and the elderly. The Oxford vaccine can be stored at normal fridge temperatures (2-8 C) for up to six months. ${ }^{10}$

The Moderna vaccine is another mRNA vaccine with a similar working principle to that of the Pfizer BioNTech vaccine. ${ }^{9}$ Its storage is much easier (remains stable up to 30 days in household fridges and up to six months at -20C).

Nadeem Zaidi FCPS, FCARCSI,

Consultant Anaesthetist, FGH.

Correspondence to: syed-nadeem.zaidi@mbht.nhs.uk 


\section{REFERENCES}

1. Riedel S. Edward Jenner and the history of small pox and vaccination. Proc (Bayl Univ Med Cent). 2005;18(1): 2125 .

2. Smith KA. Louis Pasteur, the father of immunology? Frontiers in Immunology 2012;(3):68.

3. Greenwood, B. The contribution of vaccination to global health: past, present and future. Philos Trans R Soc Lond B Biol Sci. 2014;369(1645):20130433.

4. Baicus A. History of polio vaccination. World J Virol. 2012; 1(4):108-14.

5. Plotkin S. History of vaccination. PNAS 2014; 111(34):12283-12287.

6. Pardi N, Hogan MJ, Porter FW. Weissman D. mRNA vaccines - a new era in vaccinology. Nat Rev Drug Discov. 2018;17(201): 261-279.

7. Bill RM. Recombinant protein subunit vaccine synthesis in microbes: a role for yeast? Journal pharm pharmacol. 2014;67:319-328.

8. The History of Vaccines. Website available at: https://www. historyofvaccines.org/ (accessed 02/03.21).

9. Callaway E. The race for coronavirus vaccines: A graphical guide. Nature 2020;580:576-577.

10. Polack FP, Thomas SJ, Kitchin N, Absalon J, Gurtman A, Lockhart S, et al. Safety and efficacy of the BNT162b2 mRNA Covid-19 vaccine. N Engl J Med 2020;383:26032615. 